\title{
ASSOCIAÇÃO ENTRE ATIVIDADE FÍSICA, COMPOSIÇÃO CORPORAL E APTIDÃO FÍSICA DE CRIANÇAS PRÉ-PUBERTÁRIAS
}

\author{
ASSOCIATION BETWEEN PHYSICAL ACTIVITY, BODY
} COMPOSITION AND PHYSICAL FITNESS OF PREPUBESCENT CHILDREN

ASOCIACIÓN ENTRE ACTIVIDAD FÍSICA, COMPOSICIÓN CORPORAL Y APTITUD FÍSICA DE NIÑOS PREPÚBERES

\author{
Carlos Marta (carlosmarta@ipg.pt)* | ** \\ Teresa Fonseca (tfonseca@ipg.pt)* |** \\ Bernardete Jorge (bernardete@ipg.pt)* | ** \\ António Dias (tobino@ipg.pt)* \\ Jorge Casanova (jcasanova@ipg.pt)* | ** \\ Natalina Casanova (rocasa@ipg.pt)* | **
}

\section{RESUMO}

O propósito deste estudo foi analisar a associação de diferentes índices de atividade física, na escola (IAFE), no desporto organizado (IAFD) e nos tempos livres (IAFTL), com níveis de gordura corporal e aptidão física de crianças pré-pubertárias. A amostra foi constituída por 302 crianças, 147 rapazes e 155 raparigas, de 10 e 11 anos de idade $(10,81 \pm 0,43)$. Os índices de atividade física foram avaliados através do questionário de Baecke. A avaliação da gordura corporal foi realizada pela medição de pregas subcutâneas e cálculo da percentagem de gordura corporal. Com exceção do IAFTL, verificaram-se correlações significativas e negativas dos diferentes índices de atividade física com a gordura subcutânea e percentagem de massa gorda. Verificaram-se correlações significativas e positivas: do IAFE com a capacidade aeróbia, força e resistência muscular, velocidade e agilidade; do IAFD com a capacidade aeróbia, força e resistência muscular, velocidade, agilidade e potência muscular; e do IAFTL com a capacidade aeróbia e agilidade. Estes dados poderão ajudar a compreender melhor a associação da atividade física em diferentes contextos com a aptidão física de crianças pré-púberes.

Palavras-chave: pré-púbere, performance, saúde 


\section{ABSTRACT}

The purpose of this study was to analyse the association of different physical activity indexes, in school (IAFE), organized sports (IAFD) and leisure time (IAFTL), with body fat and physical fitness levels in prepubescent children. The sample consisted of 302 children, 147 boys and 155 girls, aged between 10 and 11 years old (10.81 \pm 0.43$)$. Physical activity indexes were evaluated using the Baecke questionnaire. Skinfold thickness measurements and body fat percentage were used in the evaluation of fat mass. With the exception of the IAFTL, there were significant and negative correlations between the different physical activity indexes and the subcutaneous fat mass and body fat percentage. There were significant and positive correlations between the IAFE and the aerobic capacity, strength and muscular endurance, speed and agility; between the IAFD and the aerobic capacity, strength and muscular endurance, speed, agility and anaerobic muscular power; and between the IAFTL and the aerobic capacity and agility. These data may help to better understand the association between the physical activity in different environment and the physical fitness of prepubescent children.

\section{Keywords: prepubescent, performance, health}

\section{RESUMEN}

El propósito de este estudio fue analizar la asociación de diferentes índices de actividad física, en la escuela (IAFE), en el deporte organizado (IAFD) y en los tiempos libres (IAFTL), con niveles de grasa corporal y aptitud física de niños prepúberes. La muestra estuvo constituida por 302 niños, 147 varones y 155 niñas, con edades comprendidas entre 10 y 11 años de edad $(10,81 \pm 0,43)$. Los índices de actividad física se evaluaron a través del cuestionario Baecke. La grasa corporal se evaluó mediante la medición de los pliegues subcutáneos y el cálculo del porcentaje de grasa corporal. Con excepción del IAFTL, se verificaron correlaciones significativas y negativas de los diferentes índices de actividad física con la grasa subcutánea y el porcentaje de masa grasa. Se verificaron correlaciones significativas y positivas: del IAFE con la capacidad aeróbica, fuerza y resistencia muscular, velocidad y agilidad; del IAFD con la capacidad aeróbica, fuerza y resistencia muscular, velocidad, agilidad y potencia muscular; y el IAFTL con la capacidad 


\section{Egitania}

$s$ c i e $\Omega$ c i a

aeróbica y la agilidad. Estos datos pueden ayudar a comprender mejor la asociación de la actividad física en diferentes contextos con la aptitud física de los niños pre púberes.

Palabras clave: pre púber, rendimiento, salud

* Instituto Politécnico da Guarda

** Unidade de Investigação para o Desenvolvimento do Interior 


\section{INTRODUÇÃO}

É consentâneo que os níveis de atividade física e aptidão física em crianças e jovens se têm vindo a degradar consideravelmente nas últimas décadas (Matton et al., 2007), e que os comportamentos sedentários como ver televisão, usar o computador e jogar jogos de vídeo têm aumentado (Owen et al., 2010), com o consequente incremento dos fatores de risco para a saúde que a falta de atividade física acarreta.

Tal como a atividade física, também a aptidão física tem sido reconhecida como fator determinante para um estilo de vida saudável, referindose, cada vez mais, à aptidão física referenciada à saúde e não apenas à aptidão relacionada com o desempenho motor (Lämmle et al., 2010). Crianças com altos níveis de competência motora são mais ativas, mais aptas e gastam menos tempo em tarefas sedentária (Castelli \& Vale, 2007).

Por alguma dificuldade que existe em avaliar e quantificar níveis de atividade física separadamente, os níveis de atividade física habituais são muitas vezes considerados em conjunto (Teixeira et al., 2006). A atividade física de crianças e jovens contempla atividades realizadas em contexto escolar, lazer e desporto organizado, pelo que parece pertinente analisar a associação dos níveis de atividade física alcançada em cada um destes contextos com indicadores de aptidão física.

É comummente reconhecido o papel que desempenha o desporto organizado realizado em associações e clubes na prevenção de comportamentos inapropriados, contribuindo para a melhoria da condição física das populações em geral. Coleman et al. (2004) afirmam que, além dos clubes, muitas crianças e jovens têm apenas atividade física vigorosa nas aulas de Educação Física na escola. Mas a atividade física na escola não se restringe às aulas de Educação Física e, a esse respeito, os recreios escolares têm sido referenciados como o tempo e o espaço mais profícuos à promoção da atividade física dos alunos (USDHHS, 2000; Pereira, 2001). Eles apresentam, em relação aos tempos de atividade física orientada, a grande vantagem de proporcionarem práticas escolhidas pelas crianças, organizadas e desenvolvidas sem a intervenção dos adultos. Contudo, Bulut e Yilmaz (2008) e Neto (2008) referem que os jogos e brincadeiras realizados pelos alunos nos intervalos das aulas são cada vez em menor número, com uma forte 
tendência para atividades que não exigem a intervenção da motricidade grossa. Alguns motivos da diminuição das oportunidades para a realização destas atividades, práticas fundamentais para o desenvolvimento físico, social e mental das crianças, prendem-se com a oferta de outras atividades sedentárias, realizadas no computador ou na biblioteca, oferecidas agora às crianças em substituição das antigas práticas lúdico-desportivas de natureza física e motora (Coelho et. al., 2008). Quanto à atividade física efetuada nos tempos livres, considerando esta como sendo a que é realizada fora do âmbito escolar e dos clubes, Neto (2006) reporta que foi desaparecendo o tempo verdadeiramente livre e as atividades espontâneas e exploratórias, fruto da institucionalização e do desenvolvimento tecnológico, que fez emergir todo um conjunto de hábitos sedentários.

Neste contexto, o propósito deste estudo foi analisar a associação de diferentes índices de atividade física, na escola (IAFE), no desporto organizado (IAFD) e nos tempos livres (IAFTL), com níveis de gordura corporal e aptidão física de crianças pré-pubertárias.

\section{METODOLOGIA}

\section{AMOSTRA}

A amostra, por conveniência, foi constituída por 302 crianças, 147 rapazes e 155 raparigas, com idades compreendidas entre 10 e 11 anos de idade $(10,81 \pm 0,43)$, que frequentavam o $2^{\circ}$ Ciclo do Ensino Básico de um Agrupamento de Escolas da Guarda, centro de Portugal. Foram critérios de inclusão a inexistência de qualquer problema de saúde ou limitação física e encontrarem-se nos estádios de Tanner I-II. Os indivíduos foram cuidadosamente informados sobre o desenho do estudo e, posteriormente, os pais das crianças assinaram um documento de consentimento de participação. O estudo foi conduzido de acordo com a declaração de Helsínquia. 


\section{AVALIAÇÃO ANTROPOMÉTRICA E MORFOLÓGICA}

Todas as medições antropométricas foram realizadas de acordo com os padrões internacionais para avaliação antropométrica (Marfell-Jones et al., 2006). A massa corporal (kg) foi medida com aproximação de $0,10 \mathrm{~kg}$ utilizando uma balança digital (Seca, modelo 841. Alemanha). Para avaliar a estatura $(\mathrm{cm})$ foi usado um estadiómetro de precisão com uma escala de intervalo de 0,10 cm (Seca, modelo 214, Alemanha). Para determinar o IMC foi dividido o peso do indivíduo $(\mathrm{kg})$ pela sua altura $(\mathrm{m})$ ao quadrado. Na medição das pregas subcutâneas utilizou-se um adipómetro com escala de $0-80 \mathrm{~mm}$ e pressão constante de 10g/ mm2 (Harpenden Skinfold Caliper). O grau de fiabilidade intra-observador das medidas antropométricas não ultrapassou em nenhum caso o limite de tolerância fixado por Sobral e Silva (1997). A percentagem de gordura corporal foi calculada usando o método de Slaughter et al. (1988). O estádio de maturação, baseado nos estádios de maturação de Tanner (Tanner, 1962) foi auto-avaliado (Duke et al., 1980).

\section{AVALIAÇÃO DA ATIVIDADE FÍSICA E APTIDÃO FÍSICA}

A capacidade aeróbia (Vaivém), flexibilidade da parte inferior das costas e isquiotibiais (Senta-e-alcança), força e resistência muscular (Abdominais e extensão de braços), foram avaliados através da bateria de testes FITNESSGRAM (Meredith \& Welk, 2007). O equilibrio (Equilibrio Flamingo) foi avaliado pela bateria de testes EUROFIT (Adam et al., 1988). A agilidade $(9.14 \mathrm{~m})$ avaliou-se de acordo com a bateria de testes AAHPERD (American Alliance for Health, Physical Education, Recreation and Dance, 1976). Na avaliação da potência anaeróbia (teste de Margaria-Kalamen) foi utilizado o protocolo descrito por George et al. (1994). A força superior (lançamento de bolas medicinais) foi avaliada utilizando o protocolo descrito por Mayhew et al. (1997). O Coeficiente de Correlação Intraclasse (CCl) variou entre 0,93 e 0,99. O nível de atividade física habitual foi medido através do questionário de Baecke et al. (1982). Este questionário é composto por três partes distintas: o primeiro grupo de questões procura determinar o índice de atividade física na escola (IAFE); o segundo grupo é dedicado às atividades desporti- 
vas (IAFD); a última parte visa avaliar a atividade física nos tempos de lazer (IAFL). Cada uma destas partes forneceu os dados necessários para estimar os três índices de atividade física, cujo somatório permitiu determinar o valor da atividade física total (IAFT). O questionário é constituído por dezasseis itens, a partir dos quais se calcularam os índices atrás mencionados. As questões são fechadas e de múltipla escolha, obedecendo à escala de valores de Lickert, em que as respostas estão codificadas de 1 a 5, segundo ordem crescente de importância. A classificação das atividades foi efetuada em função do tipo de atividade, da frequência semanal, do número de meses em que se realiza e da intensidade.

\section{ANÁLISE ESTATÍSTICA}

Métodos estatísticos padrão foram utilizados para o cálculo das médias e desvios-padrão. Foi usado o Coeficiente de Correlação Intraclasse (CCl) para determinar o grau de confiabilidade entre sujeitos nos testes selecionados. A normalidade das distribuições foi verificada através do teste de Kolmogorov-Smirnov. A associação entre variáveis foi avaliada pela correlação bivariada de Pearson. A significância estatística foi aferida para $\mathrm{p} \leq 0,05$.

\section{RESULTADOS}

Após tratamento dos dados recolhidos, foram verificados os resultados presentes na tabela 1, respeitantes à avaliação das componentes antropométrica e morfológica.

Tabela 1. Descrição antropométrica e morfológica

\begin{tabular}{|l|c|c|c|c|}
\hline & Mínimo & Máximo & Média & SD \\
\hline Idade.decimal & 10,04 & 11,54 & 10,81 &, 430 \\
\hline Estatura (cm) & 124,00 & 163,80 & 144,85 & 8,18 \\
\hline Massa corporal (kg) & 23,10 & 66,65 & 40,20 & 8,80 \\
\hline Prega tricipital (mm) & 5,00 & 32,00 & 15,01 & 5,49 \\
\hline Prega subescapular (mm) & 4,00 & 31,00 & 11,13 & 6,24 \\
\hline Prega suprailíaca (mm) & 3,00 & 38,00 & 12,89 & 7,34 \\
\hline Prega abdominal (mm) & 3,00 & 38,00 & 15,07 & 7,81 \\
\hline Prega geminal (mm) & 5,00 & 38,00 & 15,29 & 6,55 \\
\hline Prega crural (mm) & 8,00 & 47,00 & 24,51 & 8,32 \\
\hline Soma skf (mm) & 31,00 & 186,00 & 93,88 & 38,44 \\
\hline
\end{tabular}




\begin{tabular}{|l|c|c|c|c|}
\hline Massa.gorda (\%) & 8,54 & 50,92 & 22,90 & 7,87 \\
\hline $\mathrm{IMC}\left(\mathrm{kg} / \mathrm{m}^{2}\right)$ & 13,64 & 28,68 & 19,04 & 3,11 \\
\hline
\end{tabular}

Os resultados obtidos na aptidão física e respetivos índices de atividades física são apresentados na tabela 2.

Tabela 2. Descrição dos testes de aptidão física e de atividade física

\begin{tabular}{|l|c|c|c|c|}
\hline & Mínimo & Máximo & Média & SD \\
\hline Vaivém $\left(\mathrm{n}^{\circ}\right)$ & 7,00 & 74,00 & 27,47 & 13,90 \\
\hline Extensão de braços $\left(\mathrm{n}^{\circ}\right)$ &, 00 & 39,00 & 10,39 & 7,65 \\
\hline Flexibilidade esquerda $(\mathrm{cm})$ & 6,00 & 30,00 & 20,65 & 6,05 \\
\hline Flexibilidade direita $(\mathrm{cm})$ & 3,00 & 30,00 & 21,30 & 5,77 \\
\hline abdominais $\left(\mathrm{n}^{\circ}\right)$ &, 00 & 75,00 & 28,86 & 17,77 \\
\hline Extensão do tronco $(\mathrm{cm})$ & 8,00 & 30,00 & 23,48 & 4,98 \\
\hline Lançamento de $3 \mathrm{~kg}(\mathrm{~cm})$ & 138,00 & 315,00 & 231,35 & 39,08 \\
\hline Lançamento de 5kg $(\mathrm{cm})$ & 112,00 & 272,00 & 186,78 & 31,06 \\
\hline Salto horizontal $(\mathrm{cm})$ & 64,00 & 197,00 & 126,97 & 24,21 \\
\hline Velocidade $(\mathrm{s})$ & 3,41 & 5,75 & 4,45 &, 46 \\
\hline Agilidade $(\mathrm{s})$ & 10,62 & 16,78 & 13,26 & 1,31 \\
\hline Equilibrio $\left(\mathrm{n}^{\circ}\right.$ faltas) &, 00 & 35,00 & 8,64 & 5,82 \\
\hline Potencia (kg-m $\left.\cdot \mathrm{s}^{-1}\right)$ & 14,96 & 89,19 & 38,35 & 13,48 \\
\hline IAFE (valor do índice) & 2,00 & 3,75 & 2,73 &, 28 \\
\hline IAFD (valor do índice) & 1,25 & 4,50 & 2,87 &, 61 \\
\hline IAFTL (valor do índice) & 1,50 & 4,25 & 3,02 &, 46 \\
\hline IAFT (valor do índice) & 3,25 & 11,25 & 8,61 & 1,01 \\
\hline
\end{tabular}

IAFE- Atividade física na escola; IAFD-Atividade física no desporto organizado; IAFTL- Atividade física nos tempos livres; IAFT- Atividade física total

Com exceção do IAFTL, verificaram-se correlações significativas e negativas dos diferentes Índices de Atividade Física com a gordura subcutânea e percentagem de massa gorda. Verificaram-se correlações significativas e positivas: (i) do IAFE com o vaivém, extensão de braços, abdominais, salto horizontal, velocidade e agilidade; do (ii) IAFD com o vaivém, extensão de braços, abdominais, lançamento da bola medicinal de 3 e 5 kg, salto horizontal, velocidade, agilidade e potência muscular; e do (iii) IAFTL com o vaivém e agilidade (tabela 3).

Tabela 3- Associação entre os Índices de Atividade Física e as variáveis antropométrica

morfológicas e de aptidão física: correlação de Pearson.

\begin{tabular}{|c|c|c|c|c|c|c|c|c|}
\hline & \multicolumn{2}{|c|}{ IAFE } & \multicolumn{2}{|c|}{ IAFD } & \multicolumn{2}{|c|}{ IAFTL } & \multicolumn{2}{|c|}{ IAFT } \\
\hline & $r$ & $\mathbf{p}$ & $r$ & p & $\boldsymbol{r}$ & p & $r$ & $\mathbf{p}$ \\
\hline soma skinfolds &,$- 154^{* * *}$ &, 007 &,$- 193^{* *}$ & ,001 &,- 037 &, 519 &,$- 212^{* * *}$ &, 000 \\
\hline$\%$ massa gorda &,$- 164^{* * *}$ &, 004 &,$- 186^{* *}$ &, 001 &,- 031 &, 589 &,$- 207^{* *}$ &, 000 \\
\hline vaivém & $219^{* * *}$ &, 000 &, $421^{* *}$ &, 000 & $146^{*}$ & ,011 &, $391^{* *}$ &, 000 \\
\hline Extensão braços & $255^{* * *}$ & 000 & $292^{* * *}$ & 000 & 051 & 373 & $297^{* *}$ &, 000 \\
\hline abdominais & $147^{*}$ & 011 & 350 ** & 000 & 066 & 255 & $299^{* *}$ &, 000 \\
\hline lançament_3kg & 020 & 728 & $227^{* *}$ &, 000 &, 013 & 824 &, $141^{*}$ &, 014 \\
\hline lançament $5 \mathrm{~kg}$ &, 002 &, 967 & $215^{* *}$ &, 000 & 038 &, 508 & $122^{*}$ &, 034 \\
\hline Salto horizontal & $135^{*}$ & 019 & $353^{* *}$ &, 000 &, 105 & 068 & $322^{* *}$ &, 000 \\
\hline
\end{tabular}




\begin{tabular}{|c|c|c|c|c|c|c|c|c|}
\hline flexibilidade $\mathrm{drt}^{a}$ & 038 &, 514 & 087 &, 129 &,- 008 & 883 & .061 & ,291 \\
\hline flexibilidade esq ${ }^{a}$ &,- 041 & 481 &, 026 & .647 &,- 025 &, 660 &,- 021 &, 715 \\
\hline extensão tronco &,- 006 &, 922 &, 004 &, 942 &,- 014 & 812 &,- 015 &, 794 \\
\hline Equilíbrio &, 033 &, 563 &,- 081 &, 159 &,- 103 & 074 &,- 085 &, 140 \\
\hline velocidade &,$- 240^{* * *}$ &, 000 &,$- 296^{* * *}$ &, 000 &,- 057 &, 323 &,$- 294^{*}$ &, 000 \\
\hline agilidade &,$- 218^{* *}$ & .000 &,$- 367^{* 4}$ &, 000 &,$- 123^{*}$ & 033 &,$- 351^{*}$ & .000 \\
\hline Potencia MK &, 016 &, 776 & $282^{* *}$ &, 000 &, 063 & 275 & $186^{* *}$ &, 001 \\
\hline
\end{tabular}

IAFE- Atividade física na escola; IAFD-Atividade física no desporto organizado; IAFTL- Atividade física nos tempos livres; IAFT- Atividade física total

DISCUSSÃO:

O propósito deste estudo foi analisar a associação de diferentes índices de atividade física com níveis de aptidão física referenciada à saúde em crianças pré-pubertárias. As crianças evidenciaram maior índice de atividade física nos tempos livres, seguido do índice de atividade física no desporto organizado e na escola. A prática desportiva foi a que melhor se associou com a aptidão física, seguida da atividade física na escola. O maior índice de atividade física registado nos tempos livres, seguido do índice de atividade física no desporto organizado e na escola, corrobora os resultados de um estudo recente com crianças de 10 e 11 anos de idade empreendido por Marta et al. (2012). Também Duke et al. (2003) avaliaram a atividade física de crianças e adolescentes entre os 9 e os 13 anos tendo registado que apenas 38.5\% participavam em atividades organizadas, enquanto $77.4 \%$ referiu participar em atividades não organizadas.

Verificamos, no entanto, que apesar do maior índice de atividade física nos tempos livres, este índice apenas se associou de forma significativa e positiva com a resistência aeróbia e a agilidade. Pelo contrário, verificaram-se associações significativas e negativas do índice de atividade física na escola e no desporto com a soma das pregas adiposas e percentagem de gordura corporal e associações positivas destes índices com a resistência aeróbia, força e resistência muscular, velocidade e agilidade. Os resultados podem surpreender se considerarmos que existe uma associação entre a atividade física na escola e a extra-escolar, porque os fisicamente ativos na escola também o são fora da escola (Williams, 1988). Estes resultados podem, no entanto, dever-se à intensidade da atividade física em cada um dos contextos estudados. Como é referido por Coleman et al. (2004) muitas crianças e jovens têm apenas atividade física vigorosa na escola e nos clubes. Para os autores, é sobretudo nas aulas de Educação Física que a atividade física é mais vigorosa. Mas a atividade física na escola não se limita às aulas de Educação Física. Com efeito, além das aulas de Educação 
Física (inseridas no horário ou consideradas nas atividades de enriquecimento curricular) e do Desporto Escolar, que surge após o $1^{\circ}$ Ciclo do Ensino Básico, os recreios constituem uma oportunidade ímpar para a realização de práticas ativas (Ridgers et al., 2005), revelando-se como cenários importantes na promoção da atividade física de intensidade moderada a vigorosa (Groffik et al., 2012).

Os resultados mostraram ainda que nenhum dos Índices de Atividade Física se associou de forma estatisticamente significativa com a flexibilidade e equilibrio. A escola é um meio excelente para implementar e sistematizar hábitos de prática desportiva e a adoção de estilos de vida saudáveis, no entanto, como refere Armstrong (1998), a familia e a comunidade em geral devem promover o jogo, a brincadeira e a recreação, elementos fundamentais para o desenvolvimento da flexibilidade e equilíbrio, como por exemplo o "jogo da macaca" ou o "jogo do elástico". Além disso, os resultados obtidos não podem ser alheios à especificidade das adaptações ao treino em idades pré-pubertárias, nomeadamente o desenvolvimento da flexibilidade, que tem revelado estar muito condicionado pelas propriedades dinâmicas dos tecidos dos tendões e constituição músculo-esquelética, bem como da capacidade de tolerância à dor (Kato et al., 2005), bem como o desenvolvimento do equilibrio, muito influenciado por diversos fatores como o género, altura, peso e constituição morfológica, que envolve múltiplos sistemas fisiológicos (neuromusculares e sensoriais), pelo que a capacidade de integrar uma série de sinais aferentes, a nível cortical, é um pré-requisito para o seu desenvolvimento (Fong et al., 2012).

Os resultados parecem sugerir que o índice de atividade física nos tempos livres, apesar de apresentar um índice maior, não se associa significativamente com níveis de gordura corporal. Contudo, relacionase significativamente com a resistência aeróbia e agilidade de crianças pré-pubertárias. Adicionalmente, sugerem a necessidade de aplicação de exercícios de flexibilidade e equilibrio em crianças neste período etário. Os resultados deste estudo permitem apoiar professores e profissionais na otimização de exercícios de perda de gordura e melhoria da aptidão física em crianças.

Este estudo apresenta algumas limitações, nomeadamente:

(i) não ter um desenho de carácter longitudinal; 
(ii) não considerar a possível influência de outras variáveis de natureza biológica, sociocultural e ambiental;

(iii) não utilizar métodos de medição objetiva na quantificação dos níveis de atividade física (ex Acelerometria).

\section{BIBLIOGRAFIA}

Adam, C., Klissouras, V., Ravassolo, M., Renson, R., Tuxworth, W., Kemper, et al. (1988). Eurofit: Handbook for the Eurofit Test of Physical Fitness. Rome: Council of Europe. Committee for the Development of Sport.

American Alliance for Health, Physical Education, Recreation and Dance (1976). Youth fitness test manual. Washington: AAHPERD

Armstrong, N. (1998). O papel da escola na promoção de estilos de vida ativos. In Sociedade Portuguesa de Educação Física e Omniserviços (eds.). A Educação para a Saúde- $O$ papel da escola na promoção de estilos de saudáveis (pp: 5-15). Lisboa.

Baecke, J., Burema, J., \& Frijiters, J. (1982). A short questionnaire for the measurement of habitual physical activity in epidemiological studies. American Journal of Clinical Nutrition, 36, 936-942.

Bulut, Z., \& Yilmaz, S. (2008). Permaculture playgrounds as a new design approach for sustainable society. International Journal of Natural and Engineering Sciences, 2, 35-40.

Castelli, D., \& Valley, J. (2007). The relationship of physical fitness and motor competence to physical activity. Journal of Teaching in Physical Education, 26, 358-374.

Coelho, R., Sousa, S., Laranjo, M.J., Monteiro, A.C., \& Bragança, G. (2008). Excesso de peso e obesidade, prevenção na escola. Ata Médica Portuguesa, 21 , 341-344.

Coleman, K., Heath, E., \& Alcala I. (2004). Overweight and aerobic fitness in children in the United States/Mexico border region. Revista Panamérica de Salud Pública, 15, 262-271.

Duke, J., Huhman, M., \& Heitzler, C. (2003). Physical activity levels among children aged 9-13 years - United States, 2002. Morbidity and Mortality Weekly Report, 52 (33), 785-788.

Duke, P.M., Litt, I.R., \& Gross, R.T. (1980). Adolescents self-assessment of sexual maturation. Pediatrics. 66(6): 918-920.

Fong, S., Fu S., \& Ng G. (2012). Taekwondo training speeds up the development of balance and sensory functions in young adolescents. Journal of Science and Medicine in Sport, 15, 64-68.

George, J., Fisher, A., \& Vehrs, P. (1994). Laboratory experiences in exercise sci- 


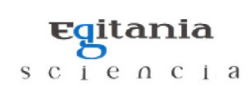

ence. Boston: Jones \& Bartlett Publishers, Inc.

Groffik, D., Sigmund, E., Fromel, K., Chmelík, F., \& Lokvencova, P.N. (2012). The contribution of school breaks to the all-day physical activity of 9-and 10-yearold overweight and non-overweight children. International Journal of Public Health, 57, $711-718$.

Kato, E., Oda, T., Chino, K., Kurihara, T., Nagayoshi, T., Fukunaga, T., \& Yasuo Y. (2005). Musculotendinous Factors Influencing Difference in Ankle Joint Flexibility between Women and Men. International Journal of Sport and Health Science, 3, 218-225.

Lämmle, L., Tittlbach, S., Oberger, J., Worth, A., \& Bös, K. (2010). Two-level model of motor performance ability. Journal of Exercise and Science \& Fitness, $8(1), 41-49$.

Marfell-Jones, M., Olds, T., Stewart, A., \& Carter, L. (2006). International standards for anthropometric assessment. Potchefstroom, South Africa: ISAK.

Marta, C., Marinho, D., Barbosa, T., Izquierdo, M., \& Marques, M. (2012). Physical fitness differences between prepubescent boys and girls. Journal of Strength \& Conditioning Research, 26(7), 1756-1766.

Mayhew, J., Ware, J. Johns, R. \& Bemben, M. (1997). Changes in upper body power following heavy-resistance strength training in college men. International Journal of Sports Medicine, 18, 516-520.

Meredith, M.D., \& Welk, G.J. (2007). FITNESSGRAM / ACTIVITYGRAM Test Administration Manual (Fourth Ed). Champaign, IL: Human Kinetics.

Neto, C. (2006). Actividade física e saúde - As políticas para a infância. Boletim do IAC, $n^{\circ} 82$, separata 20.

Neto, C. (2008). Actividade física da criança e do jovem e independência de mobilidade no meio urbano. In Pereira, B. \& Carvalho (eds.). Atividade Física Saúde e Lazer (pp: 15-34). Lousã: LIDEL.

Owen, N., Healy, G., Matthews, C. \& Dustan, D. (2010). Too much sitting: The population health science of sedentary behavior. Exercise and Sport Sciences Reviews, 3(3), 105-109.

Pereira, B. (2001). Um olhar sobre o recreio, espaço de jogo, aprendizagem e alegria mas também de conflito e medo. Violência e indisciplina na escola. Livro do colóquio, XI Colóquio AFIRSE, Lisboa: FPCE/UL.

Ridgers, N., Stratton, G., \& Fairclough, S.J. (2005). Assessing physical activity during recess using accelerometry. Preventive Medicine, 41 (1), 102-107.

Slaughter, M., Lohman, T, Boileau, R., Horswill, C., Stillman, R., VanLoan M., \& Bemben, D. (1988). Skinfold equations for estimation of body fatness in children and youth. Human Biology, 60(5), 709-723.

Sobral, F \& Silva, M. (1997). Cineantropometria: Curso Básico. Coimbra: FCDEF Tanner, J.M. (1962). Growth at adolescence. Oxford: Blackwell Scientific Publi- 


\section{Egitania}

$s$ c i e $\Omega$ c i a

cations.

Teixeira, P.J., Silva, N., Vieira N., Palmeira, L., \& Sardinha, L.B. (2006) A atividade física e o exercício no tratamento da obesidade. Endocrinologia Metabolismo e Nutrição, 15, 1-15.

USDHHS (2000). Healthy People 2010: Understanding and improving health. Washinton, DC.

Williams, A. (1988). Physical activity patterns among adolescents - some curriculum implications. Physical Education Review, 11 (1), 28-39. 